\title{
Interaction houle-courant au-dessus d'un fond de rides
}

François Marin

Maitre de conférences, LMFGC, Université du Havre

John F.A. Sleath

Reader, Laboratoire d' Hydraulique, Université de Cambridge (G B.)

Résumé

L'objectif de cette contribution est d'analyser expérimentalement l'écoulement moyen résultant de l'interaction houle-courant au-dessus d'un fond de rides. Nous avons effectué des mesures de vitesse dans un canal a houle et/ou à courant par Vélocimétrie Doppler Laser. En écoulement de courant seul, les distributions de vitesse obéissent à l'exception du voisinage immédiat du fond à une loi logarithmique caractéristique d'un écoulement turbulent. L'effet de superposer au courant une houle semble d'augmenter significativement non pas la contrainte de frottement au fond mais la rugosité équivalente de Nikuradse. Les résultats obtenus sont en bon accord avec le modele de Sleath [11].

\section{Introduction}

Quand un écoulement se propage au-dessus d'un fond meuble, des ondulations connues sous le nom de rides prennent souvent naissance sur celui-ci. Ces rides résultant de l'interaction entre l'écoulement et les sédiments peuvent être générés en écoulement stationnaire (courant seul) comme en écoulement instationnaire (houle seule ou écoulement combine houle-courant). Elles affectent de façon significative le transport sédimentaire, la dispersion de polluants, l'atténuation de la hauteur des vagues; il est donc capital d'avoir une bonne connaissance de l'écoulement au voisinage de ce type de fond.

II existe beaucoup de modeles concernant le calcul des couches limites au voisinage des fonds marins ([2]; [3]; [11]; ...). Cependant, il existe peu de résultats expérimentaux permettant de les tester, en particulier pour les fonds de rides. Le but de ce travail est d'apporter de nouvelles dcanés experimentales pour une meilleure compréhension de l'ecoulement au voisinage des fonds de rides subissant l'action combinese de la houle et du courant. 


\section{Movens experimentaux}

Les tests ont été effectués dans le canal de $17,5 \mathrm{~m}$ du Laboratoire d'Hydraulique de l'Universite de Cambridge. Dans ce canal dont la largeur interne est de $58 \mathrm{~cm}$ une houle et/ou un courant peuvent être générés. Un batteur articulé disposé à une extrémité du canal génère la houle alors qu'une plage de cailloux de pente 1:20 a l'autre extrémité l'amortit. Le courant est généré à l'aide d'une pompe travaillant en circuit fermé et délivrant un débit de 7,31 par seconde dans ce circuit. Dans cette étude la houle se propage contre le courant, l'aspiration se faisant à proximité du batteur et le refoulement au sommet de la plage d'amortissement en répartissant l'écoulement de façon uniforme sur toute la largeur du canal.

Le même fond ridé a été utilisé pour tous les tests. Il a été obtenu en faisant propager une houle au-dessus d'un fond initialement plat de sable de diamètre de grain $0,4 \mathrm{~mm}$. Des rides se sont alors formées et lorsqu'elles ont atteint leur état d'équilibre, nous les avons fixées avec une fine poudre de ciment. Les rides obtenues correspondent à des rides du type « modèle en brique » [1] caractérisées par la présence de «ponts » régulièrement répartis entre les crêtes de rides. La longueur d'onde $L$ et la hauteur $h$ de ces rides sont respectivement de $47 \mathrm{~mm}$ et $10 \mathrm{~mm}$.

La composante horizontale de la vitesse de l'écoulement a été mesurée par Vélocimétrie Doppler Laser en diffusion avant. Pour chaque test, quatre profils de vitesse ont été réalisés: deux au-dessus du sommet de ride et deux au-dessus du creux de ride, au niveau d'un pont d'une part et entre deux ponts d'autre part (Figure 1).

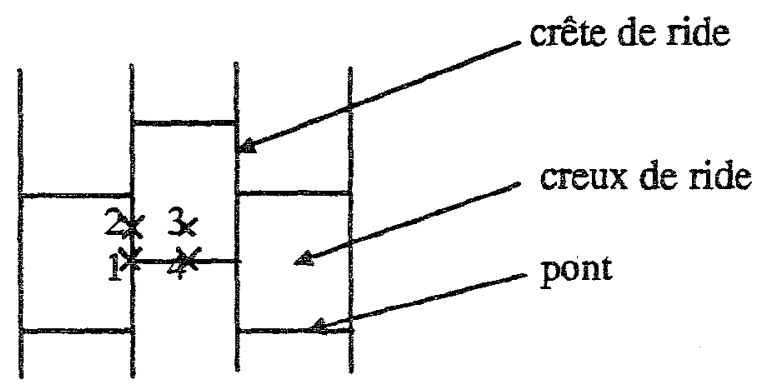

Figure 1 Localisation des profils de vitesse réalisés; 1: crête de ride, au niveau d'un pont; 2 : crête de ride, entre deux ponts; 3 : creux de ride, entre deux ponts; 4 : creux de ride, au niveau d'un pont.

La distance entre le fond et chaque point de mesure sera donnée par la coordonnée $\mathrm{Y}$ du point considéré, le niveau $\mathrm{Y}=0$ correspondant au niveau moyen du fond. Le sens positif pour les vitesses a été choisi dans le sens de propagation de la houle. Les mesures de vitesse sont réalisées en continu sur 50 périodes, chaque période étant "découpée » en 100 phases. Une vague moyenne est reconstituée et les composantes moyennes et fluctuantes de la vitesse sont alors déterminées pour chacune des phases. Un compteur réalise le traitement analogique du signal issu du photomultiplicateur; dans le cas du courant seul, le signal délivré par ce 
compteur est filtré pour les très basses fréquences $(<0,02 \mathrm{~Hz})$ afin de déterminer precisément la composante moyenne de la vitesse.

\section{Etude préliminaire: courant seul au-dessus d'un fond de rides}

Nous àvons effectué une étude préliminaire en écoulement de courant seul avant de superposer à ce courant une houle.

Les profils de vitesse moyenne obtenus au-dessus du sommet et du creux de ride sont représentés sur la figure 2, la hauteur d'eau dans le canal étant de $200 \mathrm{~mm}$.

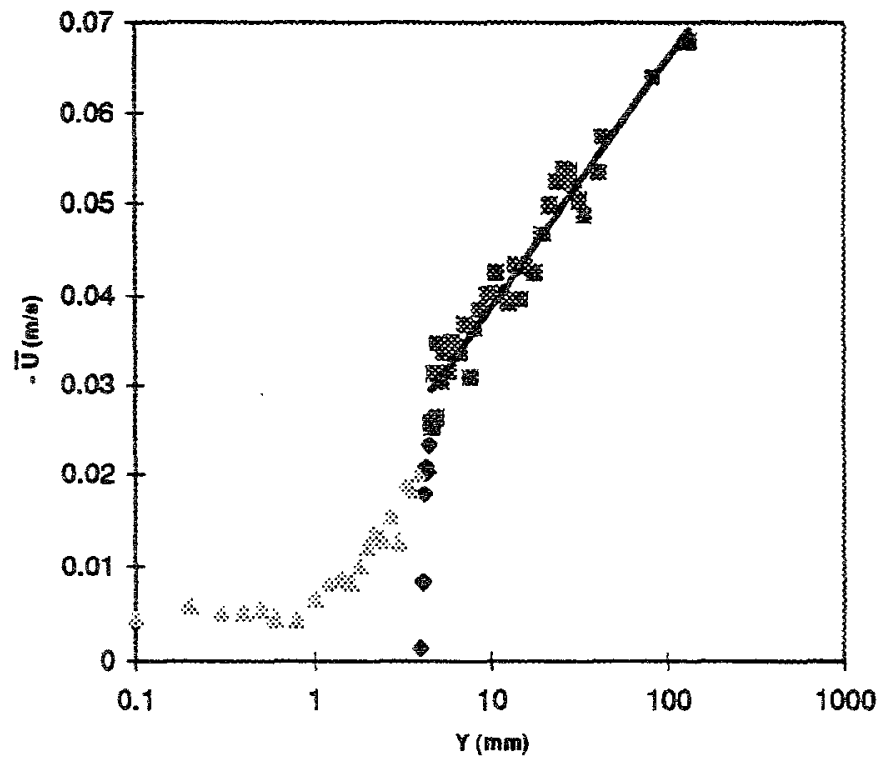

- sous-couche visqueuse

loi de paroi

creux de ride

Figure 2 Distributions des vitesses moyennes aur-dessus du sommet et du creux de ride.

Il est clair qu'en dehors des points de mesure situés au voisinage immédiat du fond, le profil de vitesse peut être décrit par une loi logarithmique caractéristique d'un écoulement turbulent. La loi qui passe au mieux par les points de mesure est donnée par l'équation suivante:

$$
-\overline{\mathrm{U}}=0,0119 \operatorname{Ln}(\mathrm{Y})+0,0113
$$

La vitesse de frottement $\mathrm{u}^{*}$ au niveau du fond peut être estimé à l'aide de la formule de Prandti-Von Karman:

$$
-\overline{\mathrm{U}}=\frac{\mathrm{u}^{*}}{\mathrm{~K}} \operatorname{Ln}\left(\frac{\mathrm{Y}}{\mathrm{Y}_{0}}\right)
$$

Nous obtenons ainsi: $\mid u *=0,0048 \mathrm{~m} / \mathrm{s} ; \mathrm{Y}_{0}=0,39 \mathrm{~mm}$ 
Nous pouvons en déduire la rugosité équivalente de Nikuradse $\mathrm{k}_{\mathrm{q}}$ du fond de rides à l'aide de la relation suivante applicable dans le cas d'une couche limite turbulente au-dessus d'un fond hydrauliquement rugueux [8]:

$$
\frac{\overline{\mathrm{U}}}{\mathrm{u}^{*}}=2,5 \mathrm{Ln}\left(\frac{\mathrm{Y}}{\mathrm{k}_{\mathrm{s}}}\right)+8,5
$$

Cette relation nous donne: $k_{z}=11,7 \mathrm{~mm}$, résultat en bon accord avec la formule (4) proposée par Van Rijn [13] qui estime $k_{s}$ à $10,9 \mathrm{~mm}$; dans cette formule, h est la hauteur de ride.

$$
\frac{\mathrm{k}_{\mathrm{s}}}{\mathrm{h}}=1,1\left(1-\mathrm{e}^{-25 \frac{\mathrm{h}}{\mathrm{L}}}\right)
$$

Un paramètre important de l'écoulement est le nombre de Reynolds suivant:

$$
\mathbf{R}_{\mathbf{I}}=\frac{\mathbf{u} * \mathbf{k}_{\mathrm{S}}}{\mathrm{v}}=58
$$

Il est traditionnellement admis que pour $\mathrm{R}_{r}<5$, le fond est hydrauliquement lisse et que pour $R_{x}>70$, le fond est hydrauliquement rugueux. Nous sommes donc dans une zone de transition où $Y_{0}$ dépend à la fois de la taille des rugosités et de l'épaisseur de la sous-couche visqueuse. Pour $R_{r}=58$, on a [9]:

$$
\frac{Y_{0}}{k_{s}}=\frac{1}{32}
$$

cette valeur est très proche de $\frac{1}{30}$, valeur admise pour un fond hydrauliquement rugueux. Ainsi, $Y_{0}=0,38 \mathrm{~mm}$, valeur en accord avec (1) et (2).

Le niveau de turbulence dans l'écoulement est tel que: $\frac{\sqrt{\mathrm{u}^{2}}}{|\mathrm{u}|}=3$ sauf au voisinage immédiat du fond où les fluctuations de vitesse diminuent très rapidement.

\section{Ecoulement combiné houle-courant: résultats et discussion}

Nous avons superposé au courant décrit dans le paragraphe précédent une houle dont nous avons fait varier l'amplitude. La houle générée dans le canal correspond pour les tests réalisés à la houle de la théorie de Stokes développée au troisième ordre [6].

Les conditions expérimentales sont données dans le tableau 1. Dans ce tableau $T$ est la période, $H$ la hauteur de la houle, d la profondeur moyenne d'eau, $k$ le nombre d'onde de la houle et $\hat{U}_{0}$ l'amplitude de la composante horizontale de la vitesse obtenue par transformée de Fourier à $40 \mathrm{~mm}$ du niveau moyen du fond. 


\begin{tabular}{|c|c|c|c|c|c|}
\hline (12s) & 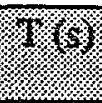 & 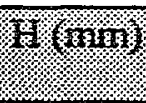 & (4) & 14 & 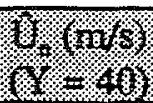 \\
\hline 1 & 1,38 & 30 & 200 & 0,74 & 0,079 \\
\hline 2 & 1,37 & 51 & 200 & 0,75 & 0,13 \\
\hline 3 & 1,38 & 61 & 200 & 0,74 & 0,17 \\
\hline
\end{tabular}

Tableau 1 Conditions expérimentales en écoulement combiné houle-courant.

Nous nous sommes limités pour cette étude à l'analyse de l'écoulement moyen. Les distributions de la vitesse moyennée sur une période de l'écoulement sont représentées sur les figures 3 .

(a)

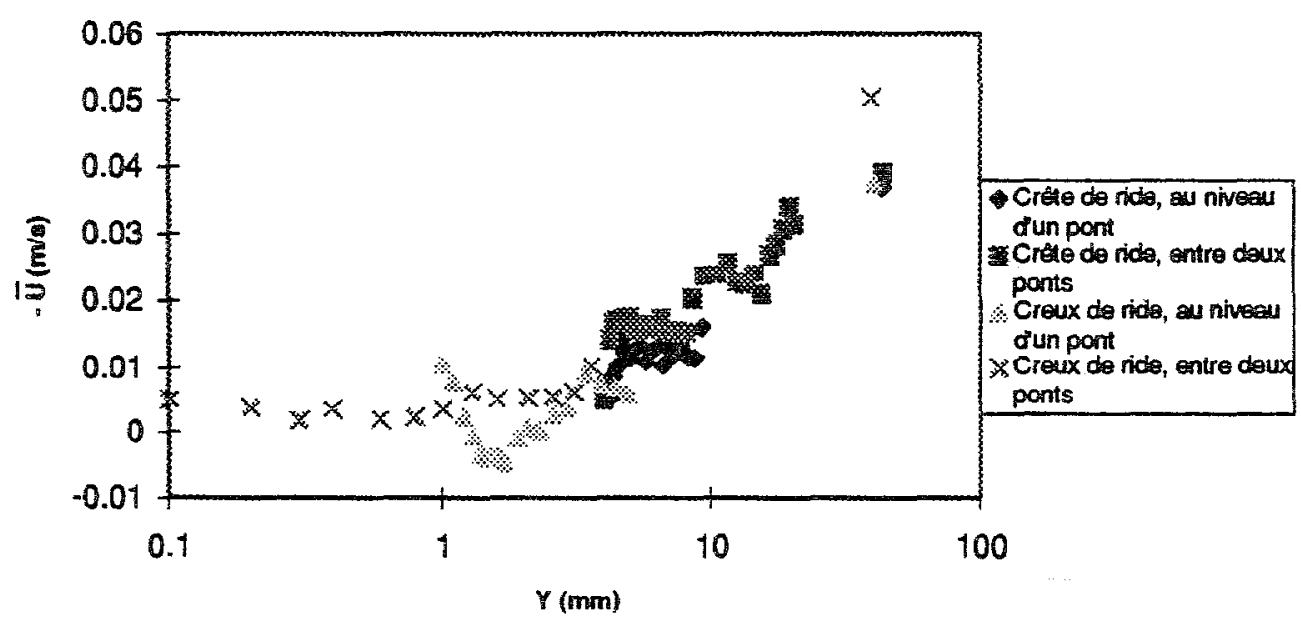

(b)

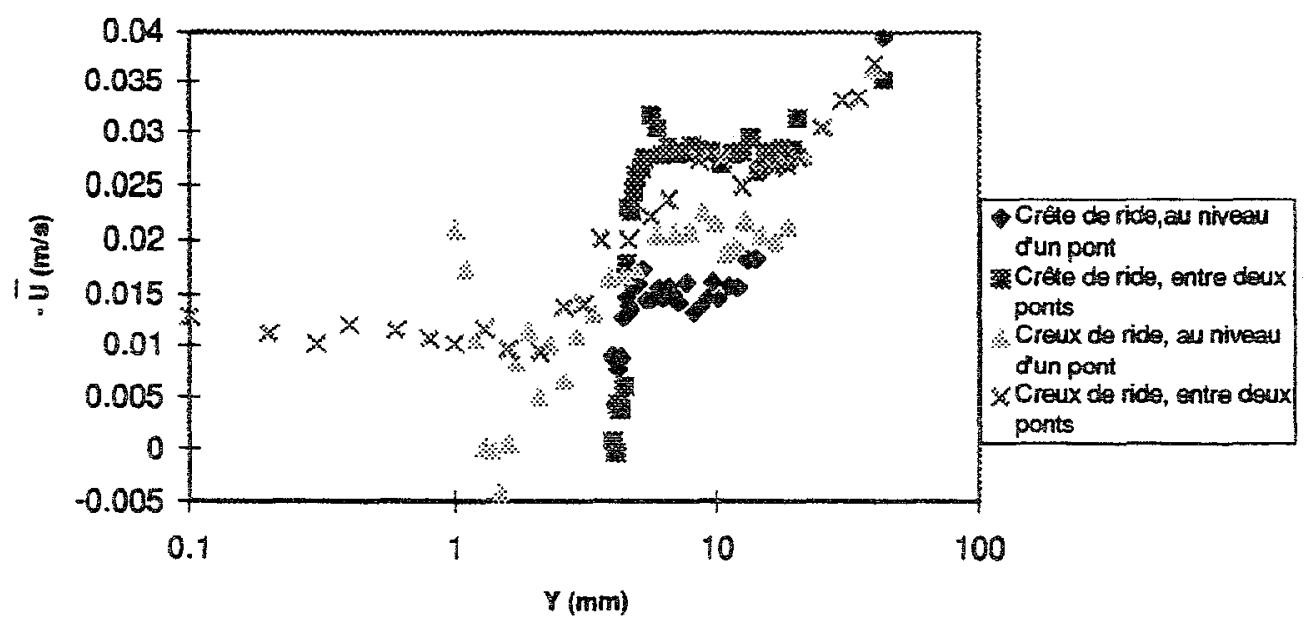


(c)

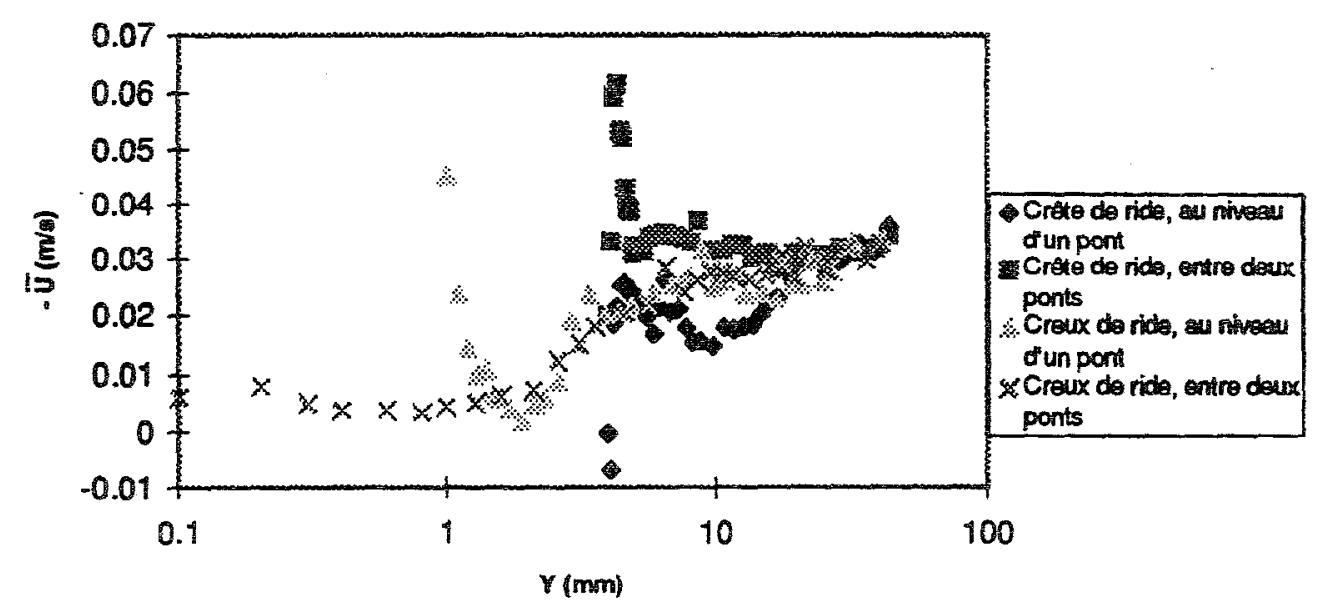

Figure 3 Distributions des vitesses moyennées sur une période de l'écoulement; (a): test $N^{\circ} 1$, (b): test $N^{\circ} 2$, (c): test $N^{\circ} 3$.

$\mathrm{Au}$ voisinage du fond, les vitesses de dérive dépendent fortement de l'endroit considéré (crête de ride entre deux ponts, creux de ride, ...). Cependant, pour chacun des tests, les différents profils de vitesse convergent plus haut vers une loi logarithmique commune. Cette loi peut être représentée par les équations suivantes:

$$
\begin{array}{ll}
\text { Test } N^{\circ} 1: & -\bar{U}=0,0116 \operatorname{Ln}(Y)-0,0045 \\
\text { Test } N^{\circ} 2: & -\bar{U}=0,012 \operatorname{Ln}(Y)-0,0085 \\
\text { Test } N^{\circ} 3: & -\bar{U}=0,012 \operatorname{Ln}(Y)-0,0107
\end{array}
$$

Kemp \& Simons [5] avait déjà montré que lorsqu'une houle se propage avec ou contre le courant, la loi logarithmique de distribution des vitesses existe encore; néanmoins, le fait de superposer une houle à un courant augmentait leur contrainte de frottement à la paroi dans le cas d'un fond rugueux alors que cette contrainte reste constante pour nos tests. La différence des conditions expérimentales utilisées peut contribuer à expliquer ce désaccord.

La superposition de la houle au courant ne semble modifier que l'échelle de rugosité $Y_{0}$ qui, en accord avec les résultats de Kemp \& Simons [5] augmente avec la hauteur de la houle. Avec (2), (7), (8) et (9), nous obtenons $Y_{0}$ respectivement égal à $1,4,2,0$ et $2,4 \mathrm{~mm}$ pour les tests $\mathrm{N}^{\circ} 1,2$ et 3 . Dans le cas d'un fond hydrauliquement rugueux, nous avons:

$$
\mathrm{Y}_{0}=\frac{\mathrm{k}_{\mathrm{s}}}{30}
$$


d'où les valeurs de $k_{:}: 43,3,60,0$ et 71,9 mm respectivement pour les tests $\mathrm{N}^{\circ} 1,2$ et 3. Dans le cas de la houle la plus forte (test $N^{\circ} 3$ ), la rugosite équivalente de Nikuradse est done six fois plus forte que dans le cas du courant seul.

Sleath [11] a développé un modèle pour déterminer les distributions de vitesse en écoulement combiné houle-courant au dessus de fonds rugueux. Dans ce modèle, le profil des vitesses moyennées sur une période de l'écoulement est donné par la relation suivante:

$$
\frac{\bar{U}}{u^{*}}=\frac{1}{K} \operatorname{Ln}\left(\frac{Y+Y_{S}}{Y_{a}}\right)
$$

avec:

$$
\begin{aligned}
& Y_{\mathrm{s}}=0,0063 \mathrm{k}_{\mathrm{s}} \frac{\mathrm{U}_{0}}{\mathrm{u}^{*}}\left(\frac{\mathrm{a}}{\mathrm{k}_{\mathrm{s}}}\right)^{\frac{1}{2}} \\
& \frac{\mathrm{Y}_{\mathrm{s}}}{\mathrm{Y}_{0}}=1+0,19 \frac{\mathrm{U}_{0}}{\overline{\mathrm{u}}^{*}}\left(\frac{\mathrm{a}}{\mathrm{k}_{\mathrm{s}}}\right)^{\frac{1}{2}}
\end{aligned}
$$

où a est l'amplitude orbitale du fluide près du fond.

La figure 4 permet de comparer nos résultats avec les résultats expérimentaux de Sleath [10], de Kemp \& Simons [4],[5], de Van Doorn [12] et le modèle de Sleath [11].

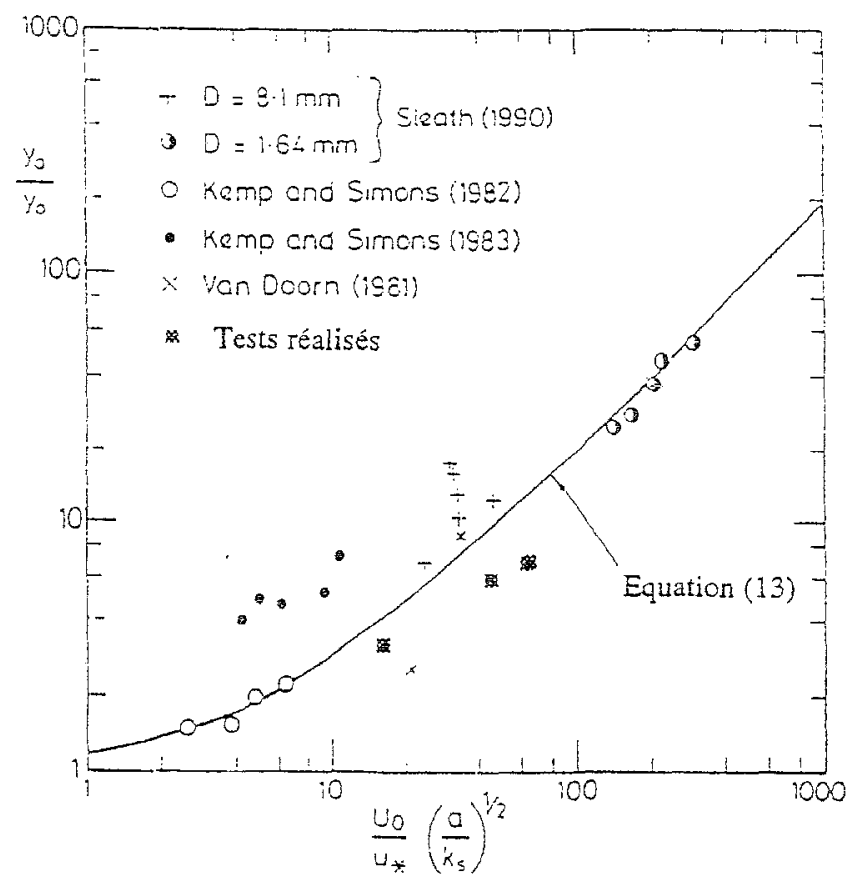

Figure 4 Evolution de $Y_{d} Y_{0}$ avec $\left(\mathrm{U}_{o} / \mathrm{u}^{*}\right)\left(a / \mathrm{k}_{\mathrm{s}}\right)^{1 / 2}$; d'après Sleath (1991), en y superposant nos résultats. 
Les résultats de Kemp \& Simons [4], [5] et de Van Doorn [12] ont été obtenus dans le cas d'un courant colinéaire à la houle, la houle se propageant dans le sens du courant pour les tests de Kemp \& Simons [4] et de Van Doorn [12]; le courant était perpendiculaire à la direction de propagation de la houle pour les tests de Sleath [10]. L'ensemble des résultats expérimentaux suit bien la courbe représentant l'équation (13) du modèle de Sleath. Nos points sont situés légèrement en-dessous de cette courbe mais il n'est pas sûr que cette différence soit significative.

Ce travail a été financé par la Communauté Européenne dans le cadre du programme « Capital Humain et Mobilité »

\section{Références}

[1] Bagnold R.A. 1946. Motion of waves in shallow water. Interaction between waves and sand bottoms. Proc. A 187 Roy. Soc., pp. 1-15.

[2] Grant W.D. et Madsen O.S. 1979. Combined wave and current interaction with a rough bottom. J. Geophys. Res., 84 (C4), pp. 1797-1808.

[3] Huynh-Thanh S. et Temperville A. 1991. A numerical model of the rough turbulent boundary layer in combined wave and current interaction. Dans: Soulsby R.L. et Bettess (Editeurs), Sand Transport in Rivers, Estuaries and the Sea. Balkema, Rotterdam, pp. 93-100.

[4] Kemp P.H. et Simons R.R. 1982. The interaction between waves and a turbulent current: waves propagating with the current. J. Fiuid Mech., Vol. 116, pp. 227-250.

[5] Kemp P.H. et Simons R.R. 1983. The interaction between waves and a turbulent current: waves propagating against the current. J. Fluid Mech., Vol. 130, pp. 73-89.

[6] Marin F. et Sleath J.F.A. 1994. Mass transport over rippled beds. Dans: Bélorgey M., Rajaona R.D. et Sleath (Editeurs), Sediment transport mechanisms in coastal environments and rivers. World Scientific, pp. 246-254.

[7] Ranasoma K.I.M. et Sleath J.F.A. 1994. Combined oscillatory and steady flow over ripples. J. Waterway, Port, Coastal, and Ocean Engineering, Vol. 120, $\mathrm{N}^{\circ} 4$, pp. 331-346.

[8] Schlichting H. 1979.Boundary-layer theory. McGraw-Hill, Seventh Edition.

[9] Sleath J.F.A. 1984. Sea bed mechanics. Wiley-Interscience.

[10] Sleath J.F.A. 1990. Velocities and bed friction in combined flows. Proc. 22nd Conf. Coastal Eng., Am. Soc. of Civ. Eng., New York, pp. 450-463.

[11] Sleath J.F.A. 1991. Velocities and shear stresses in wave-current flows. J. Geophys. Res., Vol. 96, C8, pp. 15237-15244.

[12] Van Doorn T. 1981. Experimental investigation of near bottom velocities in water waves with and without a current, Rep. M1423, Delft Hydraul. Lab., Delft, Netherlands.

[13] Van Rijn L.C. 1982. Equivalent roughness of alluvial bed. Proc. A.S.C.E. J. Hydraul. Div. 108 (HY10), pp. 1215-1218. 